www.jmscr.igmpublication.org Impact Factor 5.244

Index Copernicus Value: 83.27 ISSN (e)-2347-176x ISSN (p) 2455-0450 crossref DOI: _https://dx.doi.org/10.18535/jmscr/v4i11.19

\title{
Evaluation of Sensorineural Hearing Loss Caused by Drill Burr (Types, Size and Speed of Drilling) In Ipsilateral and Contra Lateral Ear after Middle Ear Surgery
}

\section{Authors \\ Dr Chandra Bhan ${ }^{1}$, Dr Kartikeya Purohit ${ }^{2}$, Dr Vivek Verma ${ }^{3}$, Dr Jagdish Prasad Purohit ${ }^{4}$}

${ }^{1}$ Senior Resident, Department of ENT, Head and Neck Surgery, MLB Medical College, Jhansi, UP, India

${ }^{2}$ Senior Resident, Department of ENT, Head and Neck Surgery, MLB Medical College, Jhansi, UP, India Email-kar_pur007@yahoo.com, Phone No. - +917248581321

${ }^{3}$ Senior Resident, Department of ENT, Head and Neck Surgery, MLB Medical College, Jhansi, UP, India ${ }^{4}$ Professor \& HOD, Department of ENT, Head and Neck Surgery, MLB Medical College, Jhansi, UP, India Email-dr_jpurohit@ rediffmail.com, Phone No. - +919935647631

\section{Corresponding Author}

\section{Dr Chandra Bhan}

Flat no.51, 80 PG Married Hostel, MLB Medical College, Jhansi, UP, India.

Email:- chandrabhanmsent@gmail.com, Phone No. - +919792671657, Fax- 0510-2980025

\begin{abstract}
Background and Objective- It has been observed that drilling during ear surgery can lead to noise induced sensorineural hearing loss in diseased and non-operated ear both. Hearing loss is caused by drill noise conducted to the non-operated ear by vibrations of the intact skull. This noise exposure results in dysfunction of the outer hair cells, which may produce hearing loss. Aim of our study is to evaluate the sensorineural hearing loss caused by various types of drill bit, various size of drill bit, speed of in diseased and non-operated ear during ear surgery.

Method- Study design was prospective. Hearing assessment of all patients was done by tuning fork test and pure tone audiometry and patients with only mild, moderate and moderately severe conductive hearing loss were selected for study. Patients underwent middle ear surgeries like cortical mastoidectomy, radical mastoidectomy, modified radical mastoidectomy combined approached tympanoplasty and plain tympanoplasty after taking detailed informed consent. All patients underwent post-operative hearing assessment on $10^{\text {th }}$ post-operative day and after 2 months of operation.

Result and Conclusions- Drilling at high speed (rpm) cause more sensorineural hearing loss thus according to our study best speed of drilling is $15000 \mathrm{rpm}-25000 \mathrm{rpm}$. Larger sized burr produces more sensorineural hearing loss. always avoid the use of burrs of more than $7 \mathrm{~mm}$ size. We recommend use of $<3$ mm burr. Cutting burr produces more sensorineural hearing loss than diamond burr.

Keywords- drill, sensorineural hearing loss, outer hair cell, vibration of bone.
\end{abstract}

Introduction

An estimated $2 / 3^{\text {rd }}$ of the worlds hearing impaired population are believed to be distributed among developing countries. It may be conductive, sensorineural or mixed type. Conductive hearing loss is due to defect in the middle ear while in 
case of sensorineural hearing loss there is damage to the inner earor damage to auditory nerve.

For correction of middle ear diseases and for hearing reconstruction in conductive hearing loss various type of middle ear surgeries are performed like mastoidectomies and tympanoplasties. These middle ear surgeries if done properly without damaging semicircular canals and facial nerve, without drilling near to ossicles and any other complication should result in significant improvement in hearing. After successful middle ear surgery air conduction has been found to be improved on postoperative hearing assessment by tuning fork test and pure tone audiometry. But on postoperative hearing assessment by these tests it has been found that with the improvement of air conduction many patients develop decreased bone conduction i.e. sensorineural hearing loss, not only in the operated ear but also in non-operated contralateral ear.

It is difficult to pinpoint a particular factor as responsible cause of sensorineural hearing loss during middle ear surgeries. But vibration produced by the drilling burr, type and size of drilling burr [figure-, table 6,7], noise of suction has been suspected as one of the most important factor responsible for post-operative sensorineural hearing loss ${ }^{[1]}$. Hearing loss in the contralateral ear is caused by the drill noise vibration conducted by the intact skull to the opposite ear. The noise exposure results in the dysfunction of the outer hair cells. Contralateral ear subjected to drill induced noise but spared from the surgical trauma so chance of improvement in hearing in postoperative period is more. Outer cell dysfunction may persist for more than 1 month after surgery.

\section{Observation table}

Table-1: Preoperative mean air and bone conduction threshold of diseased ear

\begin{tabular}{|l|l|l|l|l|l|}
\hline Frequency(Hz) & 250 & 500 & 1000 & 2000 & 4000 \\
\hline Mean AC threshold (dB) & $50.83 \pm 24.17$ & $64.97 \pm 24.97$ & $69.28 \pm 15.72$ & $74.05 \pm 14.05$ & $65.59 \pm 14.41$ \\
\hline Mean BC threshold(dB) & $11.30 \pm 8.70$ & $12.14 \pm 7.68$ & $14.52 \pm 5.48$ & $15.47 \pm 10.47$ & $10.95 \pm 9.05$ \\
\hline
\end{tabular}

Table-2: Preoperative mean air and bone conduction threshold of non-diseased ear

\begin{tabular}{|l|l|l|l|l|l|}
\hline Frequency(Hz) & 250 & 500 & 1000 & 2000 & 4000 \\
\hline Mean AC threshold(dB) & $19.02 \pm 10.89$ & $23.13 \pm 21.87$ & $26.38 \pm 28.62$ & $39.04 \pm 29.04$ & $40.35 \pm 30.35$ \\
\hline Mean BC threshold(dB) & $6.66 \pm 3.44$ & $8.21 \pm 6.79$ & $11.19 \pm 8.81$ & $13.80 \pm 8.80$ & $12.14 \pm 7.14$ \\
\hline
\end{tabular}

During drilling the exposed cochlea is subjected to noise levels of more than $90 \mathrm{db}$, while the contralateral cochlea to $80-85 \mathrm{db}$ and above ${ }^{[2-3]}$. Vibration of temporal bone may have implications leading to cochlear damage, and both drill and additive effect in damaging the cochlea. Cutting burr produce more noise as compared to diamond burr. Variables such as rotation speed of burr, type of burr, have been studied.

\section{Material and Methods}

The present review is a prospective study of 42 November 2015. Infection and otorrhoea were controlled before surgery by cleaning and adequate medical treatment. Detailed history about occupational noise exposure, ototoxic drug, trauma, previous ear surgery was taken. Hearing assessment of all patients was done by tuning fork test and pure tone audiometry and patients with only mild, moderate and moderately severe conductive hearing loss were selected for study. Patients with previous ear surgery, severe or Otoscopy and otomicroscopy, radiological underwent middle ear surgeries like cortical mastoidectomy, radical mastoidectomy, modified radical mastoidectomy for choleasreatoma [table2] combined approached tympanoplasty and plain tympanoplasty [table-5] after taking detailed informed consent. All patients underwent postoperative hearing assessment on $10^{\text {th }}$ postoperative day and after 2 months of operation. suction generated noise and vibration may have 
Table-3: Postoperative mean BC threshold of ipsilateral ear vs different size of burr

\begin{tabular}{|c|c|c|c|c|c|c|c|c|c|c|c|}
\hline \multirow[t]{5}{*}{ CORTICA L } & \multirow{2}{*}{$\begin{array}{l}\text { Size of } \\
\text { burr }\end{array}$} & \multicolumn{2}{|l|}{$250 \mathrm{~Hz}$} & \multicolumn{2}{|l|}{$500 \mathrm{~Hz}$} & \multicolumn{2}{|c|}{$1000 \mathrm{~Hz}$} & \multicolumn{2}{|c|}{$2000 \mathrm{~Hz}$} & \multicolumn{2}{|l|}{$4000 \mathrm{~Hz}$} \\
\hline & & $\begin{array}{l}10^{\text {th }} \\
\text { day }\end{array}$ & $\begin{array}{l}2^{\text {nd }} \\
\text { month }\end{array}$ & $\begin{array}{l}10^{\text {th }} \\
\text { day }\end{array}$ & $\begin{array}{l}2^{\text {nd }} \\
\text { month }\end{array}$ & $\begin{array}{l}10^{\text {th }} \\
\text { day }\end{array}$ & $\begin{array}{l}2^{\text {nd }} \\
\text { month }\end{array}$ & $\begin{array}{l}10^{\text {th }} \\
\text { day }\end{array}$ & $\begin{array}{l}2^{\text {nd }} \\
\text { month }\end{array}$ & $10^{\text {th }}$ day & $2^{\text {nd }}$ month \\
\hline & $1-3 \mathrm{~mm}$ & $15 \pm 00$ & $10 \pm 00$ & $20 \pm 00$ & $10 \pm 00$ & $25 \pm 00$ & $20 \pm 00$ & $20 \pm 00$ & $15 \pm 00$ & $25 \pm 00$ & $20 \pm 00$ \\
\hline & $4-6 \mathrm{~mm}$ & $25 \pm 00$ & $15 \pm 00$ & $20 \pm 00$ & $20 \pm 00$ & $35 \pm 00$ & $25 \pm 00$ & $30 \pm 00$ & $30 \pm 00$ & $35 \pm 00$ & $20 \pm 00$ \\
\hline & $7-9 \mathrm{~mm}$ & $28 \pm 03$ & $22 \pm 03$ & $28 \pm 07$ & $22 \pm 04$ & $30 \pm 10$ & $23 \pm 08$ & $33 \pm 03$ & $23 \pm 03$ & $42 \pm 07$ & $28 \pm 03$ \\
\hline \multirow[t]{3}{*}{ RADICA L } & $1-3 \mathrm{~mm}$ & $20 \pm 00$ & $15 \pm 00$ & $25 \pm 00$ & $20 \pm 00$ & $20 \pm 00$ & $20 \pm 00$ & $25 \pm 00$ & $20 \pm 00$ & $25 \pm 00$ & $15 \pm 00$ \\
\hline & $4-6 \mathrm{~mm}$ & $22 \pm 2.5$ & $17 \pm 2.5$ & $22 \pm 2.5$ & $17 \pm 2.5$ & $25 \pm 00$ & $15 \pm 00$ & $27 \pm 2.5$ & $17 \pm 2.5$ & $37 \pm 2.5$ & $30 \pm 05$ \\
\hline & 7-9 mm & $40 \pm 00$ & $35 \pm 00$ & $35 \pm 00$ & $20 \pm 00$ & $35 \pm 00$ & $25 \pm 00$ & $40 \pm 00$ & $30 \pm 00$ & $45 \pm 00$ & $40 \pm 00$ \\
\hline \multirow[t]{3}{*}{ MRM } & $1-3 \mathrm{~mm}$ & $20 \pm 00$ & $10 \pm 00$ & $25 \pm 00$ & $15 \pm 00$ & $20 \pm 00$ & $15 \pm 00$ & $25 \pm 00$ & $20 \pm 00$ & $25 \pm 00$ & $20 \pm 00$ \\
\hline & $4-6 \mathrm{~mm}$ & $30 \pm 4.4$ & $21 \pm 3.3$ & $27 \pm 03$ & $19 \pm 04$ & $28 \pm 02$ & $21 \pm 04$ & $30 \pm 05$ & $24 \pm 04$ & $35 \pm 05$ & $26 \pm 3.5$ \\
\hline & 7-9 mm & $30 \pm 00$ & $20 \pm 00$ & $30 \pm 00$ & $15 \pm 00$ & $35 \pm 00$ & $20 \pm 00$ & $35 \pm 00$ & $25 \pm 00$ & $40 \pm 00$ & $30 \pm 00$ \\
\hline \multirow[t]{3}{*}{ CAT } & $1-3 \mathrm{~mm}$ & $25 \pm 00$ & $10 \pm 00$ & $25 \pm 00$ & $20 \pm 00$ & $25 \pm 00$ & $20 \pm 00$ & $30 \pm 00$ & $15 \pm 00$ & $30 \pm 00$ & $15 \pm 00$ \\
\hline & $4-6 \mathrm{~mm}$ & $28 \pm 18$ & $22 \pm 12$ & $30 \pm 10$ & $21 \pm 06$ & $26 \pm 14$ & $23 \pm 08$ & $31 \pm 16$ & $23 \pm 13$ & $37 \pm 07$ & $24 \pm 09$ \\
\hline & 7-9mm & $35 \pm 00$ & $25 \pm 00$ & $30 \pm 00$ & $20 \pm 00$ & $30 \pm 00$ & $25 \pm 00$ & $35 \pm 00$ & $25 \pm 00$ & $45 \pm 00$ & $30 \pm 00$ \\
\hline \multirow[t]{3}{*}{ TYMPANOPLASTY } & $1-3 \mathrm{~mm}$ & $12 \pm 03$ & $12 \pm 03$ & $15 \pm 05$ & $12 \pm 03$ & $18 \pm 03$ & $12 \pm 03$ & $17 \pm 07$ & $13 \pm 02$ & $18 \pm 3.33$ & $12 \pm 03$ \\
\hline & $4-6 \mathrm{~mm}$ & $15 \pm 00$ & $05 \pm 00$ & $15 \pm 00$ & $10 \pm 00$ & $20 \pm 00$ & $15 \pm 00$ & $15 \pm 00$ & $10 \pm 00$ & $20 \pm 00$ & $10 \pm 00$ \\
\hline & $7-9 \mathrm{~mm}$ & $15 \pm 00$ & $10 \pm 00$ & $20 \pm 00$ & $15 \pm 00$ & $15 \pm 00$ & $10 \pm 00$ & $20 \pm 00$ & $15 \pm 00$ & $20 \pm 00$ & $20 \pm 00$ \\
\hline
\end{tabular}

Table-4: Postoperative mean BC threshold of contralateral ear vs different size of burr

\begin{tabular}{|c|c|c|c|c|c|c|c|c|c|c|c|}
\hline \multirow[t]{5}{*}{ CORT ICAL } & \multirow{2}{*}{$\begin{array}{l}\text { Size of } \\
\text { burr }\end{array}$} & \multicolumn{2}{|l|}{$250 \mathrm{~Hz}$} & \multicolumn{2}{|l|}{$500 \mathrm{~Hz}$} & \multicolumn{2}{|c|}{$1000 \mathrm{~Hz}$} & \multicolumn{2}{|l|}{$2000 \mathrm{~Hz}$} & \multicolumn{2}{|c|}{$4000 \mathrm{~Hz}$} \\
\hline & & $10^{\text {th }}$ day & $\begin{array}{l}2^{\text {nd }} \\
\text { month }\end{array}$ & $\begin{array}{l}10^{\text {th }} \\
\text { day }\end{array}$ & $\begin{array}{l}2^{\text {nd }} \\
\text { month }\end{array}$ & $\begin{array}{l}10^{\text {th }} \\
\text { day }\end{array}$ & $\begin{array}{l}2^{\text {nd }} \\
\text { month }\end{array}$ & $\begin{array}{l}10^{\text {th }} \\
\text { day }\end{array}$ & $\begin{array}{l}2^{\text {nd }} \\
\text { month }\end{array}$ & $\begin{array}{l}10^{\text {th }} \\
\text { day }\end{array}$ & $\begin{array}{l}2^{\text {nd }} \\
\text { month }\end{array}$ \\
\hline & $1-3 \mathrm{~mm}$ & $15 \pm 00$ & $05 \pm 00$ & $10 \pm 00$ & $05 \pm 00$ & $15 \pm 00$ & $05 \pm 00$ & $15 \pm 00$ & $10 \pm 00$ & $20 \pm 00$ & $10 \pm 00$ \\
\hline & $4-6 \mathrm{~mm}$ & $15 \pm 00$ & $05 \pm 00$ & $15 \pm 00$ & $05 \pm 00$ & $15 \pm 00$ & $10 \pm 00$ & $20 \pm 00$ & $10 \pm 00$ & $25 \pm 00$ & $15 \pm 00$ \\
\hline & $7-9 \mathrm{~mm}$ & $20 \pm 00$ & $15 \pm 00$ & $20 \pm 05$ & $15 \pm 05$ & $25 \pm 00$ & $20 \pm 10$ & $25 \pm 50$ & $20 \pm 50$ & $30 \pm 10$ & $25 \pm 05$ \\
\hline \multirow[t]{3}{*}{ RAD ICAL } & $1-3 \mathrm{~mm}$ & $15 \pm 00$ & $10 \pm 00$ & $20 \pm 00$ & $15 \pm 00$ & $15 \pm 00$ & $10 \pm 00$ & $20 \pm 00$ & $10 \pm 00$ & $20 \pm 00$ & $15 \pm 00$ \\
\hline & $4-6 \mathrm{~mm}$ & $17 \pm 2.5$ & $07 \pm 2.5$ & $17 \pm 2.5$ & $12 \pm 2.5$ & $20 \pm 50$ & $10 \pm 00$ & $22 \pm 2.5$ & $17 \pm 2.5$ & $32 \pm 2.5$ & $25 \pm 05$ \\
\hline & $7-9 \mathrm{~mm}$ & $30 \pm 00$ & $20 \pm 00$ & $30 \pm 00$ & $25 \pm 00$ & $30 \pm 00$ & $25 \pm 00$ & $35 \pm 00$ & $30 \pm 00$ & $40 \pm 00$ & $35 \pm 00$ \\
\hline \multirow[t]{3}{*}{ MRM } & $1-3 \mathrm{~mm}$ & $15 \pm 00$ & $05 \pm 00$ & $15 \pm 00$ & $05 \pm 00$ & $15 \pm 00$ & $07 \pm 00$ & $17 \pm 00$ & $12 \pm 00$ & $25 \pm 00$ & $15 \pm 00$ \\
\hline & $4-6 \mathrm{~mm}$ & $17 \pm 12$ & $705 \pm 7.5$ & $21 \pm 06$ & $10 \pm 05$ & $25 \pm 10$ & $14 \pm 09$ & $24 \pm 06$ & $22 \pm 07$ & $28 \pm 08$ & $25 \pm 04$ \\
\hline & $7-9 \mathrm{~mm}$ & $15 \pm 00$ & $10 \pm 00$ & $15 \pm 00$ & $10 \pm 00$ & $20 \pm 00$ & $15 \pm 00$ & $25 \pm 00$ & $20 \pm 00$ & $30 \pm 00$ & $25 \pm 00$ \\
\hline \multirow[t]{3}{*}{ CAT } & $1-3 \mathrm{~mm}$ & $10 \pm 00$ & $05 \pm 00$ & $15 \pm 00$ & $10 \pm 00$ & $15 \pm 00$ & $15 \pm 00$ & $20 \pm 00$ & $15 \pm 00$ & $25 \pm 00$ & $15 \pm 00$ \\
\hline & $4-6 \mathrm{~mm}$ & $23 \pm 08$ & $13 \pm 8.9$ & $26 \pm 06$ & $19 \pm 05$ & $28 \pm 13$ & $19 \pm 05$ & $25 \pm 05$ & $19 \pm 6.6$ & $31 \pm 8.7$ & $26 \pm 07$ \\
\hline & $7-9 \mathrm{~mm}$ & $25 \pm 00$ & $20 \pm 00$ & $25 \pm 00$ & $24 \pm 00$ & $25 \pm 00$ & $25 \pm 00$ & $30 \pm 00$ & $25 \pm 00$ & $35 \pm 00$ & $35 . \pm 00$ \\
\hline \multirow[t]{3}{*}{ TYMPANOPLASTY } & $1-3 \mathrm{~mm}$ & $07 \pm 03$ & $05 \pm 00$ & $08 \pm 03$ & $05 \pm 00$ & $08 \pm 03$ & $05 \pm 00$ & $11 \pm 03$ & $07 \pm 03$ & $15 \pm 05$ & $08 \pm 03$ \\
\hline & $4-6 \mathrm{~mm}$ & $05 \pm 00$ & $05 \pm 00$ & $05 \pm 00$ & $05 \pm 00$ & $10 \pm 00$ & $10 \pm 00$ & $15 \pm 00$ & $10 \pm 00$ & $20 \pm 00$ & $10 \pm 00$ \\
\hline & 7-9 mm & $10 \pm 00$ & $10 \pm 00$ & $15 \pm 00$ & $10 \pm 00$ & $15 \pm 00$ & $10 \pm 00$ & $15 \pm 00$ & $15 \pm 00$ & $25 \pm 00$ & $20 \pm 00$ \\
\hline
\end{tabular}

Table-5: Postoperative mean BC threshold of ipsilateral ear and contralateral ear vs different speed of drilling burr during surgery

\begin{tabular}{|c|c|c|c|c|c|c|c|c|c|c|c|}
\hline \multirow{2}{*}{\multicolumn{2}{|c|}{ Speed of drilling }} & \multirow{2}{*}{\multicolumn{2}{|c|}{$250 \mathrm{~Hz}$}} & \multirow{2}{*}{\multicolumn{2}{|c|}{$500 \mathrm{~Hz}$}} & \multirow{2}{*}{\multicolumn{2}{|c|}{$1000 \mathrm{~Hz}$}} & \multirow{2}{*}{\multicolumn{2}{|c|}{$2000 \mathrm{~Hz}$}} & \multirow{2}{*}{\multicolumn{2}{|c|}{$4000 \mathrm{~Hz}$}} \\
\hline & & & & & & & & & & & \\
\hline \multirow{4}{*}{$\begin{array}{l}\text { I/A } \\
\text { EAR }\end{array}$} & $15000-25000 \mathrm{rpm}$ & $21 \pm 11$ & $10 \pm 10$ & $19 \pm 06$ & $10 \pm 10$ & $21 \pm 06$ & $15 \pm 05$ & $23 \pm 13$ & $15 \pm 10$ & $27 \pm 07$ & $23 \pm 13$ \\
\hline & $25000-30000 \mathrm{rpm}$ & $25 \pm 10$ & $12 \pm 12$ & $26 \pm 06$ & $13 \pm 07$ & $24 \pm 10$ & $17 \pm 08$ & $29 \pm 11$ & $19 \pm 10$ & $31 \pm 13$ & $24 \pm 10$ \\
\hline & $30000-35000 \mathrm{rpm}$ & $26 \pm 14$ & $18 \pm 17$ & $25 \pm 15$ & $17 \pm 08$ & $27 \pm 13$ & $19 \pm 11$ & $29 \pm 13$ & $23 \pm 08$ & $34 \pm 14$ & $28 \pm 13$ \\
\hline & $35000-40000 \mathrm{rpm}$ & $32 \pm 17$ & $21 \pm 11$ & $27 \pm 12$ & $17 \pm 09$ & $28 \pm 08$ & $20 \pm 09$ & $32 \pm 17$ & $25 \pm 15$ & $37 \pm 16$ & $30 \pm 20$ \\
\hline \multirow{4}{*}{$\begin{array}{l}\text { C/L } \\
\text { EAR }\end{array}$} & $15000-25000 \mathrm{rpm}$ & $11 \pm 04$ & $06 \pm 04$ & $17 \pm 03$ & $07 \pm 03$ & $16 \pm 06$ & $07 \pm 03$ & $16 \pm 09$ & $10 \pm 05$ & $17 \pm 13$ & $15 \pm 10$ \\
\hline & $25000-30000 \mathrm{rpm}$ & $15 \pm 10$ & $06 \pm 04$ & $16 \pm 09$ & $08 \pm 07$ & $18 \pm 07$ & $10 \pm 09$ & $20 \pm 09$ & $11 \pm 09$ & $23 \pm 12$ & $16 \pm 09$ \\
\hline & $30000-35000 \mathrm{rpm}$ & $19 \pm 11$ & $11 \pm 09$ & $19 \pm 09$ & $10 \pm 09$ & $20 \pm 10$ & $13 \pm 07$ & $21 \pm 04$ & $15 \pm 10$ & $24 \pm 11$ & $16 \pm 09$ \\
\hline & $35000-40000 \mathrm{rpm}$ & $19 \pm 11$ & $10 \pm 10$ & $20 \pm 15$ & $10 \pm 10$ & $21 \pm 14$ & $14 \pm 11$ & $23 \pm 12$ & $16 \pm 14$ & $29 \pm 09$ & $22 \pm 12$ \\
\hline
\end{tabular}

Table-6: Postoperative mean BC threshold in ipsilateral and contralateral ear vs type of drill used.

\begin{tabular}{|c|c|c|c|c|c|c|c|c|c|c|c|}
\hline \multirow{5}{*}{$\mathrm{I} / \mathrm{L}$} & \multirow[t]{2}{*}{ Type of burr used } & \multicolumn{2}{|l|}{$250 \mathrm{~Hz}$} & \multicolumn{2}{|l|}{$500 \mathrm{~Hz}$} & \multicolumn{2}{|c|}{$1000 \mathrm{~Hz}$} & \multicolumn{2}{|c|}{$2000 \mathrm{~Hz}$} & \multicolumn{2}{|c|}{$4000 \mathrm{~Hz}$} \\
\hline & & $10^{\text {th }}$ day & $2^{\text {nd }}$ month & $10^{\text {th }}$ day & $\begin{array}{l}2^{\text {nd }} \text { mont } \\
\mathrm{h}\end{array}$ & $10^{\text {th }}$ day & $\begin{array}{l}2^{\text {nd }} \text { mont } \\
h\end{array}$ & $10^{\text {th }}$ day & $\begin{array}{l}2^{\text {nd }} \text { mont } \\
\mathrm{h}\end{array}$ & $10^{\text {th }}$ day & $\begin{array}{l}2^{\text {nd }} \text { mont } \\
h\end{array}$ \\
\hline & DIAMOND & $13 \pm 03$ & $12 \pm 03$ & $17 \pm 07$ & $15 \pm 05$ & $18 \pm 03$ & $16 \pm 06$ & $16 \pm 04$ & $13 \pm 08$ & $19 \pm 06$ & $12 \pm 08$ \\
\hline & CUTTING & $25 \pm 10$ & $18 \pm 08$ & $25 \pm 05$ & $19 \pm 09$ & $30 \pm 10$ & $22 \pm 07$ & $30 \pm 10$ & $23 \pm 08$ & $37 \pm 12$ & $25 \pm 15$ \\
\hline & DIAMOND+CUTTING & $20 \pm 19$ & $16 \pm 13$ & $24 \pm 15$ & $20 \pm 10$ & $26 \pm 16$ & $21 \pm 11$ & $26 \pm 17$ & $21 \pm 08$ & $34 \pm 10$ & $24 \pm 15$ \\
\hline \multirow{3}{*}{$\begin{array}{l}\mathrm{C} / \\
\mathrm{L}\end{array}$} & DIAMOND & $07 \pm 03$ & $06 \pm 04$ & $08 \pm 03$ & $06 \pm 04$ & $12 \pm 08$ & $08 \pm 07$ & $14 \pm 06$ & $10 \pm 10$ & $17 \pm 03$ & $15 \pm 05$ \\
\hline & CUTTING & $23 \pm 07$ & $12 \pm 08$ & $22 \pm 08$ & $12 \pm 08$ & $27 \pm 12$ & $19 \pm 10$ & $28 \pm 13$ & $21 \pm 09$ & $30 \pm 10$ & $21 \pm 09$ \\
\hline & DIAMOND+ CUTTING & $17 \pm 07$ & $11 \pm 06$ & $19 \pm 09$ & $12 \pm 07$ & $24 \pm 09$ & $15 \pm 09$ & $25 \pm 05$ & $20 \pm 05$ & $28 \pm 06$ & $21 \pm 09$ \\
\hline
\end{tabular}




\section{Discussion}

Noise induced hearin loss is usually a high frequency sensorineural hearing loss (SNHL), occurs when the nerves that transmit sound information from the ear do not function properly due to injury or disease. The hearing loss is typically greatest at 4,000 Hertz (Hz).

The sensorineural hearing loss after middle ear surgeries is not well established. The incidence of permanent sensorineural hearing loss after surgeries is 1.2 to $4.5 \%$ (Palva A. et. al, 1973) ${ }^{[4]}$. Multiple factors may be involved including suction, drill induced acoustic trauma of the cochlea, excess manipulation of the ossicles, and inadvertently touching the ossicles with a rotating burr but still there is lack of such studies which establish the direct relation between the causative factor and the post-operative sensorineural hearing loss.

In the present study majority of patients were male $(57.14 \%)$ with male female ratio $1.33: 1$. This is accordance to the study carried out by M.A. Yaor et. al; $2006^{[5]}$, this is due to illiteracy, less hospital attention of female patients and male dominating society in India. Choleasteatoma was found to be in $33.33 \%$ patients under study. In another study Neeraj Kasliwal et.al. ${ }^{[6]}$ found $51.6 \%$ cases of chleasteatoma while in another study by Alexandre Fernandes de azevedo et.al.Sept-Oct $2007^{[7]}$ it was found to be $37 \%$. Such a high prevalence of choleasteatoma in India is due to low socioeconomic strata in India, where people have tendency to ignore their disease for long time that gives sufficient time to choleasteatoma to evolve.

Table 1 and 2 shows preoperative hearing assessment of diseased and contralateral ear. On pure tone audiometry patients have been found to have moderate to severe grade of conductive hearing loss in the diseased ear at all frequencies with no sensorineural hearing loss was found in diseased and contralateral ear. Mean A-B gap was found to be $52.06 \mathrm{~dB}$ in the diseased ear. These finding are accordance with the study by Carrillo et.al, Dec $2007^{[8]}$, who studied audiometric findings of the patients, in which ossicular discontinuity was found intra operatively.

Table 3 shows pure tone audiometric assessment of hearing of operated ear on $10^{\text {th }}$ day and $2^{\text {nd }}$ month of postoperative period. Sensorineural hearing loss were accessed relative to type of surgery and size of burr. Maximum sensorineural hearing loss was seen in radical mastoidectomy i.e. $37.5 \mathrm{~dB}$ followed by in modified radical mastoidectomy i.e by $35 \mathrm{~dB}$, minimum sensorineural hearing loss was seen in plain tympanoplasty i.e. $16 \mathrm{~dB}$. Higher the duration of exposure of vibration produced by drill more hearing loss. Hearing loss was more at higher frequencies than lower frequencies ${ }^{[9]}$. This is supported by AA Desai et. al. July-Sep $2004^{[10]}$ who shows $30 \mathrm{~dB}$ hearing loss in case of plain tympanoplasty on $7^{\text {th }}$ post-operative day. Hearing loss was more in surgeries in which middle ear and the ossicles are manipulated excessively. Hearing loss was somewhat reversible and almost no loss remains $2^{\text {nd }}$ month postoperatively in plain tympanoplasty, while mild loss persist in case of mastoidectomies.

Table 4 shows mean BC threshold in non-operated ear on $10^{\text {th }}$ day and $2^{\text {nd }}$ months of postoperative period. It shows almost similar quantity of hearing loss in all mastoidectomy patients i.e. approx. 30 $\mathrm{db}$. Whereas almost no hearing loss is seen in cases of plain tympanoplasties. Hearing loss in contralateral ear has been found to be reversible. In cases of cortical mastoidectomies after 2 months mean $\mathrm{BC}$ threshold return to almost normal.

Table 3 and 4 shows that postoperative sensorineural hearing loss in operated and nonoperated ear on $10^{\text {th }}$ day and after 2 months. This shows that as the size of the burr increases the magnitude of postoperative sensorineural hearing loss increases for same type of surgical procedure. Hearing loss was maximum at higher frequencies i.e. at $4000 \mathrm{~Hz}$. The study was supported by Jiang D. et. al. Dec2009 ${ }^{[11]}$. Use of large burr causes more damage to the cochlea than small burr, because the larger is the diameter of the burr, greater is the vibratory force produced by the burr. 
According to P. Kylen et. al, $1997^{[2]}$ contralateral ear exposed to 5-10 dB lower noise level that of the ipsilateral ear.

Barek et al analyzed the sound intensities generated by high-speed handpieces in both the audible $(<20,000 \mathrm{~Hz})$ and ultra-sonic $(>20,000$ $\mathrm{Hz})$ frequency ranges ${ }^{[12]}$. Table 5 shows postoperative sensorineural hearing loss was minimum at 15000-25000 rpm whiles the maximum hearing loss occurred at 35000-40000 rpm [table-8]. Hearing loss was more in ipsilateral ear than contralateral ear this is probably because, as the drilling speed increases noise level produced increases along with the vibrations this was supported by the Ho-Ki Lee et.al.1999 ${ }^{[13]}$. Kilpatrick listed the sound intensities generated by high-speed handpieces (70-92 dB SPL), and lowspeed handpieces (74 dB SPL). ${ }^{[14]}$

Table 6 shows that when only diamond burr were used during surgery, there was no significant hearing loss in the ipsilateral as well as contralateral ear. Use of only cutting burr causes maximum hearing loss in both ear. This is probably because the cutting burr produces more noise level and more vibratory force. This conclusion was supported by the Parkin JL et. $a l .1980^{[3]}$, who studied the drill generated noise during surgeries and concluded that the average noise levels of drilling range from $65 \mathrm{~dB}$ to $95 \mathrm{~dB}$ varying with the drill and burr used. Cutting burr produces $09 \mathrm{~dB}$ more noise than diamond burr.

\section{Conclusions}

> Sensorineural hearing loss occurs in both ipsilateral and contralateral ear after middle ear surgery, more in operated ear due to more acoustic and vibration induced trauma during surgeries.

$>$ Postoperative sensorineural hearing loss is more at higher frequency.

$>$ Minimum postoperative sensorineural hearing loss occurs in cases of plain tympanoplasty due to minimum use of drill.

$>$ In case of plain tympanoplasty, mild postoperative sensorineural hearing loss occurs and this returns to almost normal value after 2 months.

$>$ Maximum postoperative sensorineural hearing loss occurs in radical mastoidectomy and other operative procedures involving mastoid drilling.

$>$ Drilling at high speed (rpm) cause more sensorineural hearing loss thus according to our study best speed of drilling is 15000 rpm-25000 rpm.

$>$ Larger sized burr produces more sensorineural hearing loss.always avoid the use of burrs of more than $7 \mathrm{~mm}$ size. We recommend use of $<3 \mathrm{~mm}$ burr.

$>$ Cutting burr produces more sensorineural hearing loss than diamond burr.

Conflict Of Interest- no conflict of interest

\section{References}

1. Kylen P, Arlinger S. Drill-generated noise levels in ear surgery. Acta Otolaryngol. 1976;82:402-9

2. Kylen P, Stjernvall JE, Arlinger S. Variables affecting the drill-generated noise levels in ear surgery.Acta Otolaryngol. 1977;84:252-9

3. Parkin JL, Wood GS, Wood RD, McCandless GA. Drill-andsuction generated noise in mastoid surgery. Archives of Otolaryngology. 1980; 106:926

4. Palva A, Sorri M. Can an operation of deaf ear be dangerous for hearing? Acta Otolaryngol Suppl. 1979; 360:155-7

5. M.A. Yaor, A.El-Kholy, B. Jafari; surgical management of chronic suppurative otitis media; a 3years study experience; Annals of African medicine; Vol5; No 1; 24-27, 2006

6. Neeraj Kasliwal, Snjeev Joshi, S.M. Pareek; determinants of sensorineural hearing loss in chronic ear disease; Indian journal of otolaryngology and head and neck surgery; Arch Otolaryngol; 106(2);92-96; Feb 1980 
7. Alexandre Fernandes De Azevedo, Daniele Cristine Gomes Pinto, Nicodemos Jose Alves DE Souza et al. Otorhinolaryngol; Vol 73; No 73; Sao Paulo; Sept-Oct. 2007

8. Carrillo, Ryner Jose C, Yang, Nathaniel W, Abes, Generoso T; Otology \& Neurotology; Vol 28- issue 8; pp 10341037; December 2007

9. Hegewald M, Heitman R, Wiederhold ML, Cooper JC, Gates GA. High-frequency electrostimulation hearing after mastoidectomy. Otolaryngol Head Neck Surg. 1989; 100:49-56

10. AA Desai, RG Aiyer, VK Pandya, Unnikrishnan Nair; Post oprative sensorineural hearing loss after middle ear surgery; Indian journal of otolaryngology and head and neck surgery; vol 56; no 3; july-sept 2004.

11. Dang Jiang ,Athanasios Bibas, Carlo Santuli, Neil Donnelly, George Jeronimidis, Alec Fitzgerald O Connor; The equivalent noise level generated by drilling on to the ossicular chain as measured by laser Doppler vibrometry: Atemporal bone study; The Laryngoscope; vol 117; pp 1040-1045; June 2007.

12. Barek S, Adam O, Motsch JF. Large band spectral analysis and harmful risks of dental turbines. Clin Oral Investig. 1999;3(1):49-54.

13. Ho-Ki Lee, Ek Ho Lee, Jae- Young Choi, Hong-Shink Choi, Hee-Nam Kim; Noise level of drilling instrument during mastoidectomy; Yonsei Medical Journal; vol 40; No 4; pp 339-342; 1999.

14. Kilpatrick HC. Decibel ratings of dental office sounds. J Prosthet Dent. 1981; 45 (2) :175 -178. 